\title{
BIOINFORMATICS
}

\section{Training a computer's eye on cell shape}

To map signaling networks that regulate cell morphology, researchers developed a method to quantify cell shape and integrate the resulting data to construct networks.

The microscope, one of the earliest scientific tools, allowed researchers to collect information about a cell's shape and features to begin to understand this basic unit of life. Later, by knocking out genes and observing the resulting phenotypes, researchers could identify associations between genes, moving toward the challenging goal of mapping signaling networks.

Most of these studies have been qualitative, but a group of researchers at Harvard Medical School wanted to build these genetic relationships quantitatively. In a recent article in Science, Norbert Perrimon, George Church and colleagues report a method to study the roles of individual genes in regulating cell morphology and to then use this information to describe signaling networks.

The authors performed a screen, exposing cells to one of 249 RNA interference (RNAi) or gene overexpression treatment conditions, and stochastically labeled cells in a culture
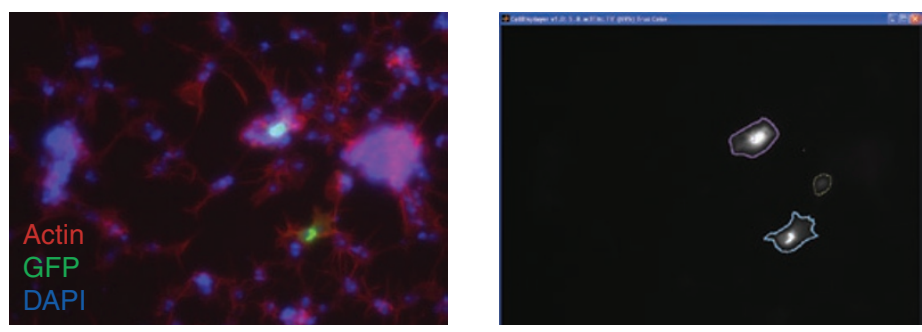

Figure 1 | Cell identification. Micrograph of cells stochastically labeled with GFP (left) and a map of the cells in the field of view identified by CellSegmenter (right). Images courtesy of Bakal and Aach.

with a fluorescent protein, which allowed them to accurately analyze several cells, rather than extracting information from all the cells in a field of view (Fig. 1).

Then they designed image-processing techniques to detect cell boundaries. "Most of the automated image-analysis programs that people use to do segmentation still weren't applicable for our needs because they approximate the boundaries and the shape," explains Chris Bakal, a postdoc in Perrimon's $\mathrm{lab}$ and co-first author on the paper with John Aach from the Church lab.

Although accurate cell boundaries could be obtained by simple intensity thresholding of fluorescent cells, the complex cell shapes and variable fluorescence often required setting a different threshold for each cell. To manage this, they developed a software application called CellSegmenter, designed by Aach, which allowed them to vary and set intensity thresholds, and select cells using simple point-and-click operations. "It's semi-automated, as we call it," says Bakal, "which is just a fancy way of saying that we do it by hand."

For each of the over 12,000 cells analyzed in this work, the cell images captured by CellSegementer were evaluated using

\section{BIOINFORMATICS}

\section{NETWORKIN IN CONTEXT-CASTING A NET FOR KINASES}

By combining sequence motifs with biological information about kinases and phosphoproteins, researchers develop an algorithm, NetworKIN, to predict in vivo phosphorylation networks.

The problem in cell biology is not so much a lack of information, but how to combine the pools of knowledge that exist. A perfect example of this is the problem of assigning specific kinases to phosphorylated proteins, which - thanks to recent developments in mass spectrometry - are being discovered with improved quality and increasing speed.

When Rune Linding, with a doctorate degree in hand for computational prediction of protein interaction motifs, joined the laboratories of Tony Pawson (Samuel Lunenfeld Research Institute) and Michael Yaffe (Massachusetts Institute of Technology), veterans in the field of phosphorylation-mediated cell signaling, the stage was set for a fruitful collaboration, resulting in NetoworKIN, an algorithm that predicts networks around phosphoproteins and their kinases.

Every kinase recognizes a specific linear motif around a serine, threonine or tyrosine. The presence of this motif, however, is not enough to unequivocally link a kinase and a protein substrate. The scientists realized that to accomplish their goal of matching kinase and substrate they would have to look beyond sequence motifs and take cellular context-for example, expression level or cellular localization—of all players into account.

Because developing a computational approach that integrates these factors from scratch would be unfeasible in terms of time and computational power, Linding sought out collaborators who could provide protein network information. His search led him to the lab of Peer Bork at the EMBL and the STRING database, which contains information on over 1.5 million interactions in almost 400 sequenced genomes. He says, "We had to do this with EMBL; no other community resource would allow you to do this, both in terms of hardware and software."

Now that they had all their collaborations lined up, the researchers developed NetworKIN; they matched phosphorylation sites with known kinase motifs and used the context network derived from STRING to arrive at the final prediction of the kinase that phosphorylates each site.

They compared the performance of their algorithm to a purely motif-based search and saw that NetworKIN 'won' hands down. Linding calculated that as much as $60-80 \%$ of substrate specificity comes from context rather than sequence motifs.

Not prone to bask in their success, Linding and Pawson already plan 
image-analysis algorithms to yield 145 morphological features. Using neural networks and clustering techniques, the researchers separated cells with similar signatures into 'phenoclusters', which then allowed them to describe a signal network that regulates cell protrusion, adhesion and tension. "The computational and statistical approach that we've applied after data extraction was what led us to some good insights," notes Bakal.

One of the goals is to study more genes using this technique, so the group is trying to automate the process by using confocal microscopy

the need for the manual correction. Bakal also envisions using this technique for small-molecule screening — generating a cell signature after treatment, and integrating it with signatures from RNAi studies to make predictions about the small molecule's targets.

This work also has potential application as a diagnostic tool. Just as scientists observe cells, pathologists examine cellular shape and features in clinical samples to determine the identity of a disease or what oncogene may be expressed. "Taking that a step further," hypothesizes Bakal, "we could make this quantitative in nature-take clinical samples, for example, assign a quantitative signature to their shape, and then by integrating that information with our database, match it up with cells overexpressing oncogenes or cells in which a tumor suppressor gene has been knocked out."

For now, CellSegmenter can be used on any image for which intensity thresholding can be used. "It's a very simple tool that allows a lot of user interface, so that simplicity allows you to do many different kinds of things with it" says Bakal.

\section{Irene Kaganman}

\section{RESEARCH PAPERS}

Bakal, C. et al. Quantitative morphological signatures define local signaling networks regulating cell morphology. Science 316, 1753-1756 (2007).

to expand NetworKIN. They want to include common interaction motifs and make the algorithm available as a webserver. Linding predicts, "people will be able to upload their proteins with the phosphorylation sites and then get predictions."

The scientists emphasize that the algorithm yields most biological insight if applied in a data-driven approach. Rather than look at a whole phosphoproteome, Linding suggests focusing on sites that are dynamically regulated in response to a certain treatment. He forsees that "NetworKIN will be very powerful for modeling when applied to a specific system."

This algorithm has the potential to be of great use to the scientific community, plans for its expansion are in place, but what is not in place yet is the funding to guarantee NetworKIN's long-term survival.

Linding and Pawson comment wistfully that it is relatively easy to start a new database, but that its maintenance is more difficult. "It is also a question of political will," Linding says; "there is very little support to maintaining things, but when you want to do really good science, you need to maintain the databases and keep them current all the time."

Let us hope that those exercising the political will agree. Nicole Rusk

\section{RESEARCH PAPERS}

Linding, R. et al. Systematic discovery of in vivo phosphorylation networks. Cell 129, 1415-1426 (2007).

\section{NEWS IN BRIEF}

\section{SPECTROSCOPY}

\section{Looking at fast kinetic events by NMR}

Although NMR spectroscopy is a fantastic technology for obtaining detailed information about proteins at the atomic level, it has been limited to studying kinetic processes on the order of minutes to hours. Schanda et al. now introduce SOFAST NMR spectroscopy, which reduces the time resolution to a few seconds. They used the SOFAST method to observe folding of $\alpha$ lactalbumin and unfolding of ubiquitin.

Schanda, P. et al. Proc. Natl. Acad. Sci. USA 104, 11257-11262 (2007).

\section{STEM CELLS}

\section{Large-scale human stem cell characterization}

As a service to the community, the International Stem Cell Initiative has systematically characterized 59 independently derived human embryonic stem cell lines from 17 different laboratories. Remarkably, in testing the expression of 17 marker cell-surface antigens and 93 genes, they found common expression patterns in spite of the differences in genetic backgrounds and different protocols used.

The International Stem Cell Initiative, Nat. Biotechnol. 25, 803-816 (2007).

\section{IMAGING AND VISUALIZATION}

\section{Triplex molecular beacons}

Molecular beacons are useful probes for analyzing nucleic acids. In their free state they form a quenched stem-loop structure, but upon binding a target sequence the stem-loop opens, restoring fluorescence. Grossman et al. describe the design of 'triplex' molecular beacons, which bind an additional stem-forming oligonucleotide sequence in their free state. Their modular assembly allows the introduction of other functionalities, such as an additional quencher.

Grossman, T.N. et al. Angew. Chem. Int. Ed. 46, 5223-5225 (2007).

\section{BIOPHYSICS}

\section{Making porous vesicles}

The encapsulation of biomolecules inside lipid bilayer vesicles offers a way to observe molecular interactions for extended periods. Expanding the capabilities of the technique, Cisse et al. describe methods for introducing pores into vesicles, allowing exchange of buffer and chemical conditions in a controlled manner while keeping the local protein concentration constant. The system shows promise for use in single-molecule experiments. Cisse, I. et al. Proc. Natl. Acad. Sci. USA; published online 11 June 2007.

\section{CHEMICAL BIOLOGY}

\section{Testing peptide permeability}

Tools for modulating protein activity, cyclic peptides are more proteolytically resistant than their linear counterparts, and their constrained conformation may provide more favorable binding characteristics and potentially increase cell-permeability. Kwon and Kodadek used a reporter gene assay to quantitatively compare cell permeability, and found that cyclic peptides are not generally more permeable than linear peptides. Kwon, Y.U. \& Kodadek, T. Chem. Biol. 14, 671-677 (2007). 